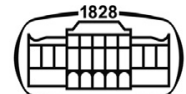

AKADÉMIAI KIADÓ

Journal of Behavioral Addictions

9 (2020) 4, 1011-1021

DOI:

$10.1556 / 2006.2020 .00092$

(c) 2020 The Author(s)

\section{FULL-LENGTH REPORT}

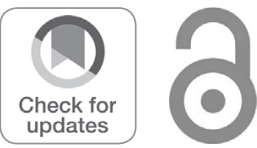

\title{
Effects of transcranial direct current stimulation on addictive behavior and brain glucose metabolism in problematic online gamers
}

\author{
HYEONSEOK JEONG ${ }^{1,2}$, JIN KYOUNG $\mathrm{OH}^{1}$,
} EUN KYOUNG CHOI ${ }^{1}$, JOOYEON JAMIE IM ${ }^{1}$, SUJUNG YOON ${ }^{3}$, HELENA KNOTKOVA ${ }^{4,5}$, MAROM BIKSON ${ }^{6}$, IN-UK SONG ${ }^{7}$, SANG HOON LEE ${ }^{8 *} \odot$ and YONG-AN CHUNG ${ }^{1,2 * *}$ (1)

\author{
${ }^{1}$ Department of Nuclear Medicine, Incheon St. Mary's Hospital, College of Medicine, The Catholic \\ University of Korea, Seoul, South Korea \\ ${ }^{2}$ Department of Radiology, Incheon St. Mary's Hospital, College of Medicine, The Catholic \\ University of Korea, Seoul, South Korea \\ ${ }^{3}$ Department of Brain and Cognitive Sciences and Ewha Brain Institute, Ewha Womans University, \\ Seoul, South Korea \\ ${ }^{4}$ MJHS Institute for Innovation in Palliative Care, New York, NY, USA \\ ${ }^{5}$ Department of Family and Social Medicine, Albert Einstein College of Medicine, The Bronx, NY, USA \\ ${ }^{6}$ Department of Biomedical Engineering, The City College of New York, New York, NY, USA \\ ${ }^{7}$ Department of Neurology, Incheon St. Mary's Hospital, College of Medicine, The Catholic \\ University of Korea, Seoul, South Korea \\ ${ }^{8}$ Department of Radiology, Yeouido St. Mary’s Hospital, College of Medicine, The Catholic \\ University of Korea, Seoul, South Korea
}

Received: January 28, 2020 • Revised manuscript received: August 11, 2020; October 15, 2020 • Accepted: October 30, 2020 Published online: December 23, 2020

\begin{abstract}
Background and aims: Some online gamers may encounter difficulties in controlling their gaming behavior. Previous studies have demonstrated beneficial effects of transcranial direct current stimulation (tDCS) on various kinds of addiction. This study investigated the effects of tDCS on addictive behavior and regional cerebral metabolic rate of glucose (rCMRglu) in problematic online gamers. Methods: Problematic online gamers were randomized and received 12 sessions of either active $(n=13)$ or sham tDCS $(n=13)$ to the dorsolateral prefrontal cortex over 4 weeks (anode F3/cathode F4, $2 \mathrm{~mA}$ for $30 \mathrm{~min}, 3$ sessions per week). Participants underwent brain ${ }^{18} \mathrm{~F}$-fluoro-2-deoxyglucose positron emission tomography scans and completed questionnaires including the Internet Addiction Test (IAT), Brief Self-Control Scale (BSCS), and Behavioral Inhibition System/Behavioral Activation System scales (BIS/BAS) at the baseline and 4-week follow-up. Results: Significant decreases in time spent on gaming $(P=0.005)$, BIS $(P=0.03)$, BAS-fun seeking $(P=0.04)$, and BAS-reward responsiveness $(P=0.01)$, and increases in BSCS $(P=0.03)$ were found in the active tDCS group, while decreases in IAT were shown in both groups $(P<0.001)$. Group-by-time interaction effects were not significant for these measures. Increases in BSCS scores were correlated with decreases in IAT scores in the active group $(\beta=-0.85, P<0.001)$. rCMRglu in the left putamen, pallidum, and insula was increased in the active group compared to the sham group ( $P$ for interaction $<0.001)$. Discussion and conclusions: $\mathrm{tDCS}$ may be beneficial for problematic online gaming potentially through changes in self-control, motivation, and striatal/insular metabolism. Further larger studies with longer follow-up period are warranted to confirm our findings.
\end{abstract}

E-mail: leesangh@catholic.ac.kr

${ }^{* *}$ Corresponding author.

E-mail: yongan@catholic.ac.kr

\section{KEYWORDS}

problematic online gaming, transcranial direct current stimulation, prefrontal cortex, positron emission tomography, regional cerebral metabolic rate of glucose 


\section{INTRODUCTION}

Online gaming has become one of the most popular Internet activities and it can have positive effects on emotion and cognition if not abused (Granic, Lobel, \& Engels, 2014). While most game players do not develop problems, some players encounter difficulties in controlling game use and show addiction symptoms (Han et al., 2011). Furthermore, problematic online gaming may lead to Internet gaming disorder (IGD), which has been included as a preliminary clinical condition in the fifth edition of the Diagnostic and Statistical Manual of Mental Disorders (DSM-5) (American Psychiatric Association, 2013). In comparison to nonproblematic engagement in gaming, IGD is characterized by compulsion, intrusive thoughts, and irresistible urge to play online games, and therefore has negative influences on mental health and daily functioning (Ho et al., 2014; Pawlikowski \& Brand, 2011). However, there has been a controversy surrounding the concept and diagnostic criteria of IGD such as withdrawal and tolerance, and thus more research is needed to reach a general consensus (Kuss, Griffiths, \& Pontes, 2017).

Various treatment strategies including psychotherapy and pharmacological intervention have been proposed for treating problematic online gaming or IGD. Although previous studies have reported the efficacy of cognitive-behavioral therapy and antidepressant medication in IGD patients (Han, Hwang, \& Renshaw, 2010; Wolfling et al., 2019), their benefits remain to be further established (King et al., 2017).

Transcranial direct current stimulation (tDCS) modulates brain function by applying a low-intensity direct current to the scalp. In general, the anodal stimulation depolarizes the neurons and facilitates the occurrence of action potentials, whereas the cathodal stimulation hyperpolarizes the neurons and suppresses the likelihood of action potentials (Nitsche et al., 2008). Compared to transcranial magnetic stimulation, tDCS has lower spatial resolution, but it is less expensive, easier, and safer to administer (Bikson et al., 2016).

The prefrontal-striatal pathway is closely implicated in the pathophysiology of various neuropsychiatric disorders including addiction (Kober et al., 2010). Previous neuroimaging studies in IGD have consistently reported structural and functional abnormalities in this circuit (Yao et al., 2017). Particularly, the dorsolateral prefrontal cortex (DLPFC) plays an important role in many aspects of cognitive control in addiction. The neurocognitive model on modulating the DLPFC in addiction suggests that the stimulatory effects can spread over not only other prefrontal regions such as the orbitofrontal cortex and ventromedial prefrontal cortex, but also the limbic and paralimbic structures including the striatum, insula, amygdala, and hippocampus through the frontal-subcortical networks (Bonelli \& Cummings, 2007; Fecteau, Fregni, Boggio, Camprodon, \& Pascual-Leone, 2010). Moreover, dopamine release is also facilitated in the prefrontal-striatal circuit (Cho \& Strafella, 2009; Rudroff, Workman, Fietsam, \& Ponto, 2020; Strafella, Paus, Barrett,
\& Dagher, 2001). These neural cascades may have influences on cognitive systems including decreases in reward seeking, impulsivity, craving, and addiction-relevant memory and attentional biases, and increases in inhibitory control (Fecteau et al., 2010). Several studies have provided evidence that $\mathrm{tDCS}$ to the DLPFC can be effective in treating various kinds of substance and behavioral addictions (Boggio et al., 2008; Fregni et al., 2008; Sauvaget et al., 2015).

Positron emission tomography (PET) has unique potential for assessing cerebral metabolism, neuroreceptor occupancy, and neurotransmitter binding in vivo. In particular, mapping uptake of ${ }^{18} \mathrm{~F}$-fluoro-2-deoxyglucose (FDG) provides insight into the brain activity patterns. Several PET studies have investigated brain pathophysiology of addiction including IGD (Tian et al., 2014) and drug dependence (Wiers, Cabrera, Skarda, Volkow, \& Wang, 2016). However, it has been sparsely used in tDCS studies compared with other neuroimaging modalities (Rudroff et al., 2020). We previously reported changes in regional cerebral metabolic rate of glucose (rCMRglu) associated with tDCS in online gamers (Lee et al., 2018) and patients with mild cognitive impairment (Yun, Song, \& Chung, 2016) and Alzheimer's disease (Im et al., 2019).

Our single-arm feasibility study in online gamers demonstrated the possibility that tDCS to the DLPFC may reduce both addiction symptoms and time spent on games (Lee et al., 2018, 2019). Based on these results, we performed a randomized, single-blind, sham-controlled trial of prefrontal tDCS in problematic online gamers using the same tDCS protocol. The main objective was to investigate whether addiction symptoms and time spent on games would decrease after tDCS. The secondary objective was to examine changes in self-control and motivation, both of which are associated with online game addiction (Kim et al., 2016) and may be partially modulated by tDCS (Loftus, Yalcin, Baughman, Vanman, \& Hagger, 2015; Ohmann, Kuper, \& Wacker, 2018). In addition, we examined associations between changes in self-control or motivation and changes in addiction symptoms or time spent on games. The third objective was to assess changes in rCMRglu to examine the neural correlates of tDCS using FDG-PET, and to explore correlations between rCMRglu and clinical characteristics.

\section{METHODS}

\section{Participants}

Young adults of age $\geq 20$ years who play online games and were interested in changing their gaming behavior were recruited from the local community. We defined the inclusion criteria for problematic online gamers as scores of 40 or higher on the Internet Addiction Test (IAT) according to the previous studies (Tsitsika, Critselis, Janikian, Kormas, \& Kafetzis, 2011; Young, 1998). Exclusion criteria were major medical conditions including neurological or psychiatric disorders; taking psychotropic medication; history of 
traumatic brain injury; history of seizure or epilepsy; and pregnancy. None of the participants had received tDCS or other medical treatment for their gaming behavior in the past. In addition, participants were paid for their participation.

\section{Study protocol}

After screening, participants underwent baseline clinical and neuroimaging evaluations and were randomized into active or sham tDCS groups. Within 1 week after the baseline assessment, participants started the course of 12 active or sham tDCS sessions at the hospital over 4 weeks ( 3 times per week). Within 1 week after the last tDCS session, follow-up clinical and neuroimaging assessments were performed using the same protocol as the baseline assessment.

\section{Transcranial direct current stimulation}

The $\mathrm{tDCS}$ parameters were based on our preliminary study (Lee et al., 2018, 2019) and the conventional protocols for human studies (Thair, Holloway, Newport, \& Smith, 2017). tDCS was applied via two sponge electrodes $(6 \mathrm{~cm}$ in diameter) using the YDS-301N device (YBrain Inc, South Korea). Each sponge was soaked with approximately $6 \mathrm{~mL}$ of saline solution $(0.9 \% \mathrm{NaCl})$. The anodal and cathodal electrodes were positioned over the left (F3, 10-20 system) and right DLPFC (F4), respectively. Bihemispheric stimulation may induce stronger cortical activations (Kwon \& Jang, 2012; Lindenberg, Nachtigall, Meinzer, Sieg, \& Floel, 2013) and bifrontal tDCS has been widely used to modulate behavior and cognition and to treat neuropsychiatric disorders including addiction (Kekic, Boysen, Campbell, \& Schmidt, 2016). The current intensity was $2 \mathrm{~mA}$ and duration of each session was $30 \mathrm{~min}$ based on the recommended safety thresholds (Thair et al., 2017). For the active sessions, the current was ramped up to $2 \mathrm{~mA}$ over the first $30 \mathrm{~s}$, remained at $2 \mathrm{~mA}$ for $29 \mathrm{~min}$, and ramped down to $0 \mathrm{~mA}$ over the last $30 \mathrm{~s}$. For the sham condition, the current was ramped up to $2 \mathrm{~mA}$ over $30 \mathrm{~s}$ and ramped down over next $30 \mathrm{~s}$. This initial and brief stimulation is the most commonly used and reliable technique for sham tDCS by mimicking cutaneous sensations of active tDCS (Gandiga, Hummel, \& Cohen, 2006). Participants were planned to be excluded from the analysis if they received less than 10 out of $12 \mathrm{tDCS}$ sessions.

\section{Clinical assessment}

The presence of mental disorders was screened by the Structured Clinical Interview for DSM-IV (First, Spitzer, Gibbon, \& Williams, 2002). The severity of gaming addiction was assessed using the IAT (Lee et al., 2013; Young, 1998) that was modified to specify online gaming instead of general online activities. In addition, participants reported average weekly hours spent on gaming over 4 weeks. Levels of self-control were evaluated with the Brief Self-Control Scale (BSCS), with higher scores indicating stronger selfcontrol (Hong, Kim, Kim, \& Kim, 2012; Tangney,
Baumeister, \& Boone, 2004). The Behavioral Inhibition System/Behavioral Activation System (BIS/BAS) scale was used to assess individual differences in the sensitivity of two motivational systems (Carver \& White, 1994; Kim \& Kim, 2001). The BIS scale examines anxiety to anticipated punishment. The drive subscale of the BAS scale evaluates constant pursuit of desired goals. The BAS-fun seeking subscale assesses how much one craves and seeks out new rewards impulsively. The BAS-reward responsiveness subscale reflects positive emotional responses to rewards. Smoking status and regular alcohol drinking (defined as at least once a week) were checked by self-report questions.

\section{Image acquisition and analysis}

Brain FDG-PET scans was performed using a Discovery STE PET-CT scanner (GE Healthcare, Milwaukee, WI, USA) at baseline and follow-up assessments. All participants were injected with 185-222 MBq of FDG intravenously and rested in a supine position with eyes closed in a quiet and dark room for $45 \mathrm{~min}$. A total of 47 transaxial emission images were obtained (pixel size $=1.95 \times 1.95 \mathrm{~mm}$, slice thickness $=3.27 \mathrm{~mm}$, matrix $=128 \times 128$ ). Computed tomography (CT) images were also acquired for attenuation correction. Standard filtering techniques and ordered subset expectation maximization algorithm were applied to reconstruct PET images.

Statistical Parametric Mapping 12 (SPM; Wellcome Department of Cognitive Neurology, Institute of Neurology, London, UK) was used for image processing and analysis. All PET images were spatially normalized to the standard PET template, resliced with a voxel size of $2 \times 2 \times 2 \mathrm{~mm}$, and smoothed with an $8 \mathrm{~mm}$ full-width at half-maximum isotropic Gaussian kernel. Relative FDG uptake at each voxel was estimated as a ratio to the global mean uptake using proportional scaling.

To compare changes in rCMRglu after tDCS between the active and sham groups, group-by-time interaction effects were examined across the whole brain in a voxel-wise manner. The statistical threshold was $P<0.001$ and 50 or more contiguous voxels. For each significant cluster, individual rCMRglu values were extracted using MarsBar toolbox (http://marsbar.sourceforge.net/).

\section{Statistical analysis}

For sample size estimation, there are no published shamcontrolled tDCS studies in gamers yet, to the best of our knowledge. From our single-arm feasibility study of tDCS in gamers (Lee et al., 2018), the estimated sample size was 9 for uncontrolled design based on the effect size of changes in IAT scores $(d=1.07)$, a power of 0.8 , and a two-tailed alpha of 0.05 . In addition, two sham-controlled tDCS studies in gambling disorder were conducted for 16 and 10 participants per group with 1 and 3 sessions, respectively (Dickler et al., 2018; Soyata et al., 2019). Considering different study designs, sample characteristics, and tDCS protocols among these studies, we aimed to recruit 13 individuals for each group. 
Table 1. Baseline characteristics of study participants ${ }^{\mathrm{a}}$

\begin{tabular}{|c|c|c|c|}
\hline Characteristics & $\begin{array}{c}\text { Active tDCS } \\
(n=13)\end{array}$ & $\begin{array}{l}\text { Sham tDCS } \\
\quad(n=13)\end{array}$ & Test \\
\hline \multicolumn{4}{|l|}{ Demographic characteristics } \\
\hline Age (years) & $\begin{array}{c}22.2 \pm 1.7 \\
(20-26)\end{array}$ & $\begin{array}{c}23.2 \pm 1.6 \\
(20-26)\end{array}$ & $P=0.16^{\mathrm{c}}$ \\
\hline Sex (male/female) & $8 / 5$ & $7 / 6$ & $P=0.69^{\mathrm{d}}$ \\
\hline Higher education $^{\mathrm{b}}$ & 11 & 11 & $P=1.00^{\mathrm{d}}$ \\
\hline Right handedness & 12 & 13 & $P=1.00^{\mathrm{d}}$ \\
\hline \multicolumn{4}{|l|}{ Behavioral characteristics } \\
\hline Years of gaming & $\begin{array}{c}5.0 \pm 3.0 \\
(1-10)\end{array}$ & $\begin{array}{c}6.0 \pm 3.5 \\
(1-14)\end{array}$ & $P=0.43^{c}$ \\
\hline IAT & $\begin{array}{c}49.2 \pm 7.9 \\
(40-66)\end{array}$ & $\begin{array}{c}49.4 \pm 7.2 \\
(40-63)\end{array}$ & $P=0.96^{\mathrm{c}}$ \\
\hline Average weekly hours spent on gaming & $\begin{array}{c}16.1 \pm 11.0 \\
(7-42)\end{array}$ & $\begin{array}{c}18.3 \pm 8.4 \\
(7.5-35)\end{array}$ & $P=0.34^{\mathrm{e}}$ \\
\hline BSCS & $\begin{array}{c}32.5 \pm 5.3 \\
(25-43)\end{array}$ & $\begin{array}{c}35.6 \pm 6.1 \\
(25-43)\end{array}$ & $P=0.17^{\mathrm{c}}$ \\
\hline BIS & $\begin{array}{c}20.9 \pm 3.1 \\
\quad(13-25)\end{array}$ & $\begin{array}{c}19.8 \pm 3.8 \\
(14-28)\end{array}$ & $P=0.54^{c}$ \\
\hline BAS-drive & $\begin{array}{c}11.2 \pm 1.3 \\
(9-14)\end{array}$ & $\begin{array}{c}11.8 \pm 2.0 \\
(8-15)\end{array}$ & $P=0.37^{\mathrm{c}}$ \\
\hline BAS-fun seeking & $\begin{array}{c}11.8 \pm 2.2 \\
(8-15)\end{array}$ & $\begin{array}{c}12.2 \pm 1.6 \\
(9-14)\end{array}$ & $P=0.61^{\mathrm{c}}$ \\
\hline BAS-reward responsiveness & $\begin{array}{c}17.1 \pm 1.8 \\
(14-20)\end{array}$ & $\begin{array}{c}16.2 \pm 1.4 \\
(14-19)\end{array}$ & $P=0.20^{c}$ \\
\hline Smoking & 5 & 6 & $P=1.00^{\mathrm{d}}$ \\
\hline Regular alcohol drinking ${ }^{\mathrm{f}}$ & 10 & 10 & $P=1.00^{\mathrm{d}}$ \\
\hline
\end{tabular}

BAS: Behavioral Activation System; BIS: Behavioral Inhibition System; BSCS: Brief Self-Control Scale; IAT: Internet Addiction Test; tDCS: transcranial direct current stimulation.

${ }^{a}$ Data are presented as mean \pm standard deviation (range) or $n$.

${ }^{\mathrm{b}}$ College or higher.

${ }^{c}$ Independent $t$-test.

d Fisher's exact test.

e Mann-Whitney U test.

${ }^{\mathrm{f}}$ At least once a week.

Normality of the data was tested with Shapiro-Wilk test. Baseline demographic and behavioral characteristics were compared using independent $t$-test, Mann-Whitney U test, or Fisher's exact test, if appropriate.

Between-group differences of changes in behavioral characteristics including time spent on games and scores of IAT, BSCS, BIS/BAS were assessed by linear mixed model. Group, time, and group-by-time interaction terms were included as fixed effects and within-subject factor was considered as a random effect. In addition, within-group changes were examined for each group using separate linear mixed model analyses.

Linear regression or Spearman's rank correlation were used to evaluate associations between significant changes in time spent on gaming or IAT scores and significant changes in BSCS or BIS/BAS scores in each group. Furthermore, linear regression or Spearman's rank correlation were used to investigate relationships between rCMRglu and behavioral characteristics in the entire sample at baseline and between significant changes in rCMRglu and significant changes in behavioral characteristics within the active tDCS group.
A $P$ value less than 0.05 (two-tailed) was considered statistically significant. Statistical tests were performed using STATA version 16 (StataCorp., College Station, TX, USA).

\section{Ethics}

This study was approved by the Institutional Review Board of Incheon St. Mary's Hospital (Incheon, South Korea) and carried out in accordance with the Declaration of Helsinki. All participants provided written informed consent.

\section{RESULTS}

\section{Characteristics of study participants}

A total of 26 problematic online gamers were enrolled and randomized into active $(n=13)$ or sham tDCS groups $(n=$ 13) (Supplementary Fig. 1). No participants were dropped from the study or analysis due to withdrawal of consent, follow-up loss, or low compliance. There were no significant differences in the number of tDCS sessions received between the two groups $(P=0.72)$ and no participants reported 
adverse events from tDCS. There were no significant differences between the two groups in any baseline characteristics including age $(P=0.16)$, sex $(P=0.69)$, years of gaming $(P=0.43)$, average weekly hours spent on gaming $(P=0.33)$, and IAT score $(P=0.96)$ (Table 1$)$.

\section{Effects of tDCS on behavioral characteristics}

Changes in behavioral characteristics are demonstrated in Table 2. Group-by-time interaction effects were not significant for any of the measures. However, the active tDCS group showed significant decreases in average weekly hours spent on gaming $(P=0.005)$ and scores on IAT $(P<0.001)$, BIS $(P=0.03)$, BAS-fun seeking $(P=0.04)$, and BASreward responsiveness $(P=0.01)$, and increases in BSCS score $(P=0.03)$. The IAT score was also significantly decreased in the sham tDCS group $(P<0.001)$.

Results from the correlation analysis among the changes of behavioral characteristics in the active tDCS group are demonstrated in Supplementary Table 1. Increases in the BSCS scores were associated with decreases in the IAT scores in the active group $(\beta=-0.85, P<0.001)$. However, a significant correlation was not found in the sham tDCS group $(\beta=-0.24, P=0.43)$ (Fig. 1).

\section{Effects of tDCS on brain glucose metabolism}

A significant group-by-time interaction effect was found in a cluster encompassing the left putamen, pallidum, and insula $(P<0.001)$ (Table 3 and Fig. 2). Post-hoc tests revealed that rCMRglu was increased in the active tDCS group $(P<$ $0.001)$, while it was reduced in the sham tDCS group $(P<$ $0.001)$.
Results from the correlation analysis between rCMRglu and behavioral characteristics are presented in Supplementary Tables 2 and 3. At the baseline, rCMRglu in the abovementioned cluster had negative relationships with scores of BAS-fun seeking $(\beta=-0.54, P=0.004)$ and BASreward responsiveness $(\beta=-0.57, P=0.002)$ in the entire sample (Fig. 3).

\section{DISCUSSION}

This study investigated the effects of repeated tDCS to the DLPFC in problematic online gamers. Our findings indicate that active tDCS may be associated with reductions in time spent on online gaming, increases in self-control, and changes in motivation, while addiction severity was decreased in both active and sham groups. In addition, rCMRglu of the putamen, pallidum, and insula was enhanced in the active group compared to the sham group. These results suggest that prefrontal tDCS may be effective for improving control of gaming behaviors and cerebral glucose metabolism of striatal-insular structures.

Our findings on decreased addiction severity and time spent on games and increased self-control after active tDCS were consistent with the findings from our previous preliminary study (Lee et al., 2018). Furthermore, decreases in the IAT scores were associated with increases in self-control only in the active group. Low self-control is one of the core features of addiction (Lee et al., 2012; Reynolds, Penfold, \& Patak, 2008). Previous studies reported lower self-control in IGD and smartphone addiction than healthy controls (Kim et al., 2016; $\mathrm{Na}$, Lee, Choi, \& Kim, 2017). In addition, the scores of BIS,

Table 2. Changes of behavioral characteristics after transcranial direct current stimulation ${ }^{\mathrm{a}}$

\begin{tabular}{|c|c|c|c|c|c|c|c|}
\hline \multirow[b]{3}{*}{ Characteristics } & \multicolumn{3}{|c|}{ Active tDCS } & \multicolumn{3}{|c|}{ Sham tDCS } & \multirow{3}{*}{$\begin{array}{c}\text { Test } \\
(\text { group } \times \text { time })\end{array}$} \\
\hline & \multicolumn{3}{|c|}{$(n=13)$} & \multicolumn{3}{|c|}{$(n=13)$} & \\
\hline & Baseline & Follow-up & Test (time) ${ }^{\mathrm{b}}$ & Baseline & Follow-up & Test (time) $)^{\mathrm{b}}$ & \\
\hline IAT & $\begin{array}{c}49.2 \pm 7.9 \\
(40-66)\end{array}$ & $\begin{array}{l}35.8 \pm 15.5 \\
(17-66)\end{array}$ & $\begin{array}{l}P<0.001 \\
d=1.00\end{array}$ & $\begin{array}{l}49.4 \pm 7.2 \\
(40-63)\end{array}$ & $\begin{array}{l}31.4 \pm 12.9 \\
\quad(2-52)\end{array}$ & $\begin{array}{l}P<0.001 \\
d=1.40\end{array}$ & $\begin{array}{l}P=0.36 \\
d=0.35\end{array}$ \\
\hline $\begin{array}{l}\text { Average weekly hours spent } \\
\text { on gaming }\end{array}$ & $\begin{array}{l}16.1 \pm 11.0 \\
\quad(7-42)\end{array}$ & $\begin{array}{c}9.4 \pm 6.9 \\
(0.5-23)\end{array}$ & $\begin{array}{c}P=0.005 \\
d=0.75\end{array}$ & $\begin{array}{c}18.3 \pm 8.4 \\
(7.5-35)\end{array}$ & $\begin{array}{l}16.3 \pm 10.5 \\
(2-40)\end{array}$ & $\begin{array}{l}P=0.34 \\
d=0.25\end{array}$ & $\begin{array}{l}P=0.13 \\
d=0.56\end{array}$ \\
\hline BSCS & $\begin{array}{c}32.5 \pm 5.3 \\
(25-43)\end{array}$ & $\begin{array}{r}35.8 \pm 7.1 \\
(24-45)<\end{array}$ & $\begin{array}{l}P=0.03 \\
d=0.58\end{array}$ & $\begin{array}{l}35.6 \pm 6.1 \\
(25-43)\end{array}$ & $\begin{array}{c}36.6 \pm 6.7 \\
(23-45)\end{array}$ & $\begin{array}{l}P=0.44 \\
d=0.21\end{array}$ & $\begin{array}{l}P=0.25 \\
d=0.44\end{array}$ \\
\hline BIS & $\begin{array}{c}20.7 \pm 3.1 \\
(13-25)\end{array}$ & $\begin{array}{l}19.6 \pm 2.6 \\
(14-24)\end{array}$ & $\begin{array}{l}P=0.03 \\
d=0.57\end{array}$ & $\begin{array}{c}19.8 \pm 3.8 \\
(14-28)\end{array}$ & $\begin{array}{c}19.0 \pm 4.5 \\
(10-24)\end{array}$ & $\begin{array}{l}P=0.31 \\
d=0.27\end{array}$ & $\begin{array}{l}P=0.81 \\
d=0.09\end{array}$ \\
\hline BAS-drive & $\begin{array}{l}11.2 \pm 1.3 \\
\quad(9-14)\end{array}$ & $\begin{array}{c}10.9 \pm 1.0 \\
(10-13)\end{array}$ & $\begin{array}{l}P=0.48 \\
\mathrm{~d}=0.19\end{array}$ & $\begin{array}{l}11.8 \pm 2.0 \\
(8-15)\end{array}$ & $\begin{array}{l}11.8 \pm 2.2 \\
\quad(9-16)\end{array}$ & $\begin{array}{l}P=0.91 \\
d=0.03\end{array}$ & $\begin{array}{l}P=0.69 \\
d=0.15\end{array}$ \\
\hline BAS-fun seeking & $\begin{array}{l}11.8 \pm 2.2 \\
(8-15)\end{array}$ & $\begin{array}{l}10.8 \pm 2.2 \\
\quad(9-15)\end{array}$ & $\begin{array}{l}P=0.04 \\
d=0.55\end{array}$ & $\begin{array}{l}12.2 \pm 1.6 \\
(9-14)\end{array}$ & $\begin{array}{l}11.8 \pm 1.8 \\
\quad(9-15)\end{array}$ & $\begin{array}{l}P=0.39 \\
d=0.23\end{array}$ & $\begin{array}{l}P=0.35 \\
d=0.35\end{array}$ \\
\hline BAS-reward responsiveness & $\begin{array}{l}17.1 \pm 1.8 \\
(14-20)\end{array}$ & $\begin{array}{c}15.8 \pm 2.2 \\
(11-19)\end{array}$ & $\begin{array}{l}P=0.01 \\
d=0.66\end{array}$ & $\begin{array}{c}16.2 \pm 1.4 \\
(14-19)\end{array}$ & $\begin{array}{c}15.5 \pm 1.1 \\
(13-17)\end{array}$ & $\begin{array}{l}P=0.07 \\
d=0.48\end{array}$ & $\begin{array}{l}P=0.48 \\
d=0.27\end{array}$ \\
\hline
\end{tabular}

BAS: Behavioral Activation System; BIS: Behavioral Inhibition System; BSCS: Brief Self-Control Scale; IAT: Internet Addiction Test; tDCS: transcranial direct current stimulation.

${ }^{a}$ Data are presented as mean \pm standard deviation (range).

${ }^{b}$ Time effects were assessed by linear mixed model in each group.

${ }^{c}$ Group-by-time interaction effects are evaluated using linear mixed model. 
A

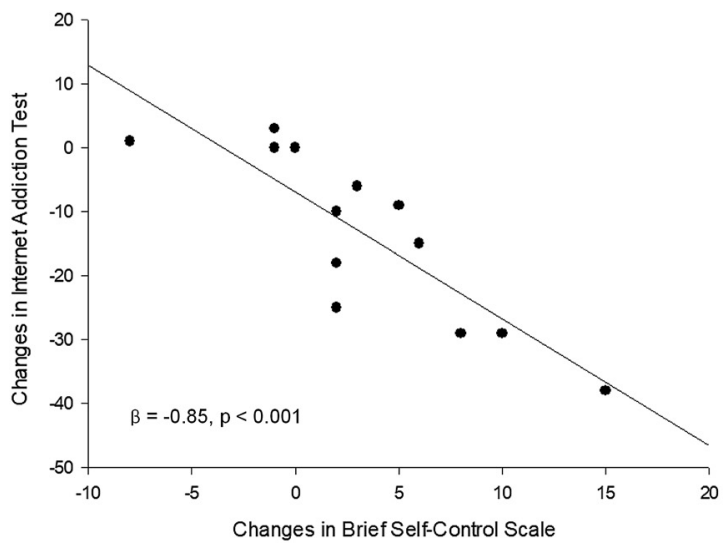

B

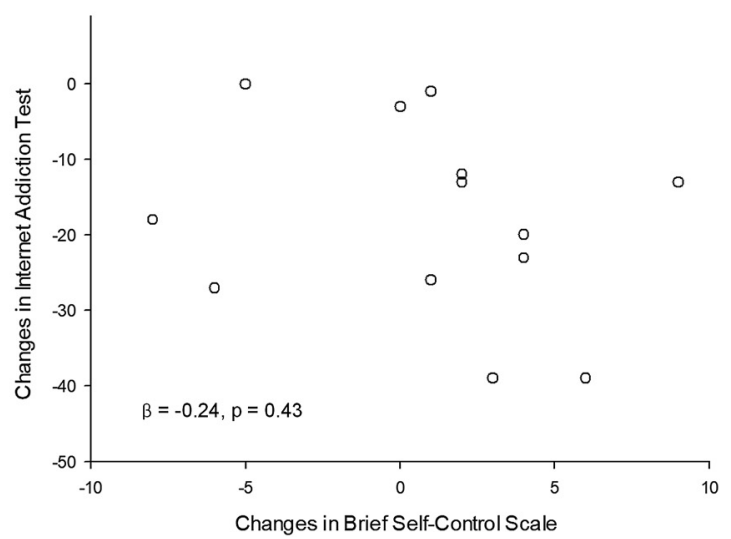

Fig. 1. Relationships between changes in the Brief Self-Control Scale and changes in the Internet Addiction Test in problematic online gamers administered with (A) active or (B) sham tDCS. A solid line represents a regression line. tDCS: transcranial direct current stimulation

Table 3. Changes of brain glucose metabolism after transcranial direct current stimulation

\begin{tabular}{lcccc}
\hline Region & $t$ & $P$ & Coordinates $^{\mathrm{a}}(x, y, z)$ & Cluster size (voxels) \\
\hline $\begin{array}{l}\text { Group-by-time interaction } \\
\text { Left putamen, pallidum, and insula }\end{array}$ & 5.47 & $<0.001$ & $-28,-12,-6$ & 159 \\
\hline
\end{tabular}

${ }^{a}$ The coordinates refer to the Montreal Neurological Institute coordinate system.

A

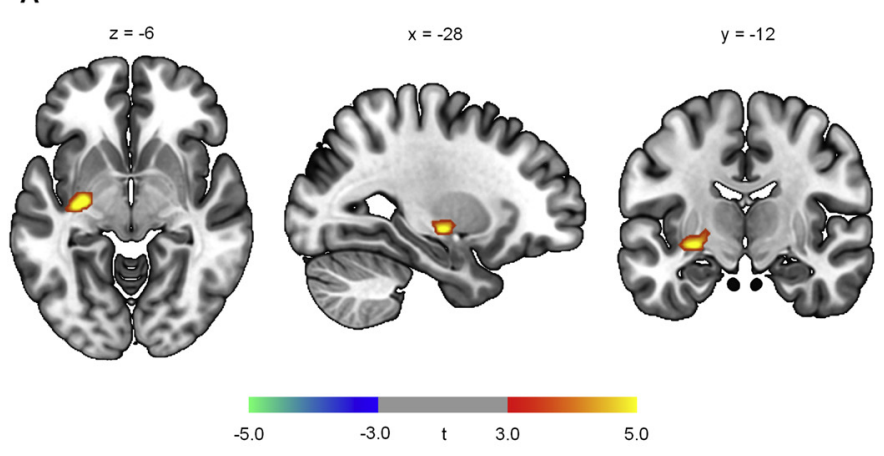

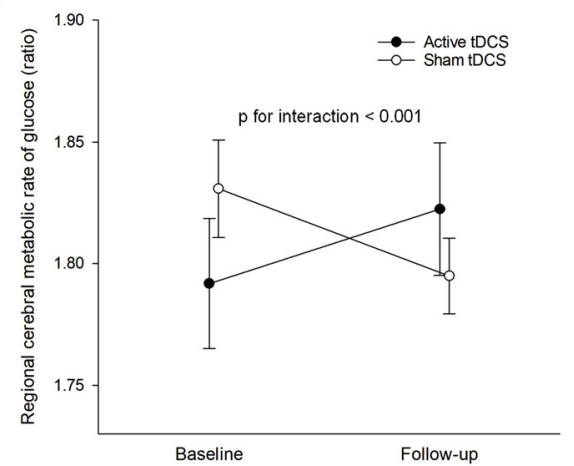

Fig. 2. Increases (red-yellow) or decreases (blue-green) of rCMRglu in problematic online gamers after active tDCS compared to sham tDCS. (A) A significant group-by-time interaction effect was found in the left putamen, pallidum, and insula $(P<0.001)$. The significant cluster was overlaid on a standard T1-weighted template and brain regions were identified using the Harvard-Oxford Atlas. Images are shown in neurological convention and the numbers above the brain slices indicate standard coordinates in the Montreal Neurological Institute space. (B) Changes in rCMRglu in the significant cluster are demonstrated for each group. Post-hoc tests revealed that rCMRglu was increased in the active tDCS group $(P<0.001)$, while it was decreased in the sham tDCS group $(P<0.001)$. Error bars represent standard errors. rCMRglu: regional cerebral metabolic rate of glucose; tDCS: transcranial direct current stimulation

BAS-fun seeking, and BAS-reward responsiveness were reduced following active tDCS. Previous studies indicated higher scores of BIS/BAS in individuals with Internet or smartphone addiction (Kim et al., 2016; Ko et al., 2008; Yen, Ko, Yen, Chen, \& Chen, 2009). Individuals with both higher sensitivity to reward stimuli and higher anxiety to anticipated punishment may choose online gaming due to lower risk and immediate pleasure. Anonymity, faceless interaction, lack of physical harm, entering or leaving in games without limitation may allow the user to feel relief of anxiety from their behaviors and to prefer online gaming to real-world activities (Yen et al., 2009). Increases of self-control and changes of motivation following tDCS may contribute to amelioration of problematic online gaming. 
A

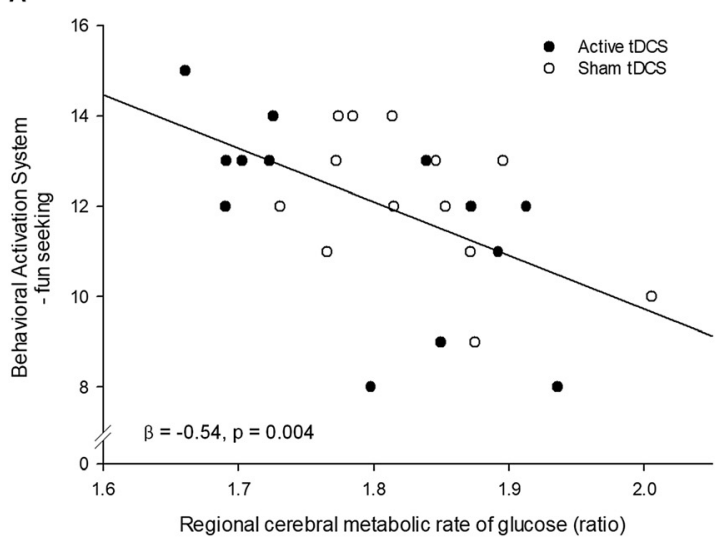

B

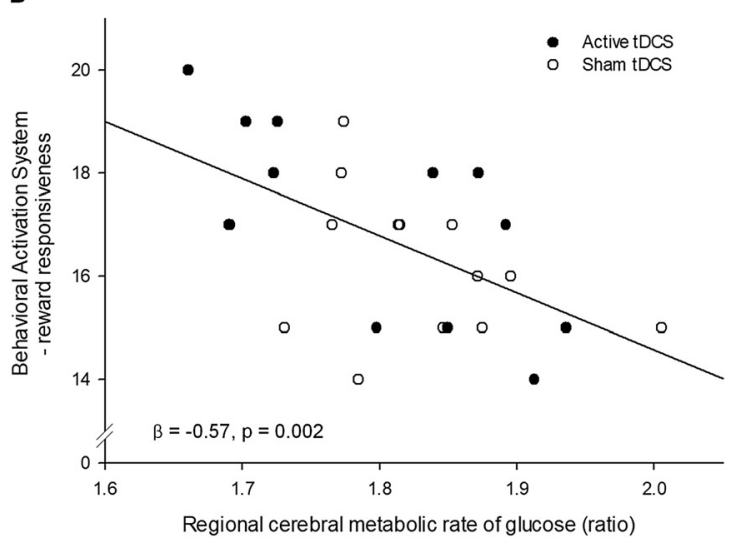

Fig. 3. Associations between regional cerebral metabolic rate of glucose in the cluster encompassing the left putamen, pallidum, and insula and (A) fun seeking and (B) reward responsiveness subscales of the Behavioral Activation System at baseline. Solid lines represent regression lines

The decreased addiction severity in the sham tDCS group was probably due to placebo effects. Attaching tDCS electrodes to the scalp itself may result in expectation of significant effects. Some studies in major depression also reported comparable antidepressant effects of sham tDCS to active stimulation (Loo et al., 2010; Palm et al., 2012). However, the amount of gaming time was not significantly changed in the sham group, although the relatively large variance should be considered in the interpretation of our findings.

Since group-by-time interaction effects were not significant for behavioral measures, efficacy of tDCS should be confirmed in further larger studies. In addition, problematic gaming is often accompanied by mood disturbances and substance abuse (Van Rooij et al., 2014), and thus assessing changes in these comorbid symptoms would be useful in future studies. Indeed, tDCS has been suggested to reduce depressive symptoms and craving for alcohol and nicotine (Boggio et al., 2008; Fregni et al., 2008; Kekic et al., 2016).

Active tDCS enhanced rCMRglu in the striatal and insular regions compared to sham tDCS. Neuroimaging and computational modelling studies have suggested that effects of tDCS are not limited to the stimulation sites, but spread to other cortical and subcortical structures. For instance, changes of rCMRglu, regional cerebral blood flow, and resting-state functional connectivity were found in both local and remote brain regions after tDCS, indicating brainwide network effects of tDCS (Keeser et al., 2011; PenaGomez et al., 2012; Rudroff et al., 2020). Although the complex mechanisms of action remain to be further elucidated, previous human neuroimaging studies revealed that bifrontal tDCS elevated both glutamate/glutamine and dopamine levels in the striatum (Fonteneau et al., 2018; Hone-Blanchet, Edden, \& Fecteau, 2016). Since tDCS alters glutamatergic activities under the electrodes (Stagg et al., 2009), stimulation to the prefrontal cortex may impact striatal activity through the corticostriatal pathways.
Previous human neuroimaging studies have suggested potential associations between gaming addiction and structural, functional, and neurochemical alterations of the striatum and insula. At resting condition, lower FDG uptake of the putamen and the negative correlation between rCMRglu of the insula and addiction severity were found in individuals with IGD (Kim et al., 2019). Another PET study in IGD demonstrated lower rCMRglu of the putamen and posterior insula, and the association between decreased dopamine $\mathrm{D} 2$ receptors of the putamen and higher addiction severity while playing online games (Tian et al., 2014). Adolescents with IGD showed reduced resting-state functional connectivity between the putamen and posterior insula, suggesting impaired network disturbances (Hong et al., 2015). Among structural neuroimaging studies, gray matter loss of the putamen and insula was consistently reported in IGD (Weinstein, 2017; Yao et al., 2017). In addition, a greater amount of videogame play in children was associated with not only damaged white matter microstructures crosssectionally but also the progression of these deficits in several brain areas including the putamen, pallidum, and insula (Takeuchi et al., 2016). The correlation between lower rCMRglu of the striatal/insular areas and higher levels of fun seeking and reward responsiveness found in this study may also imply potential roles of these deficits in problematic online gaming.

The striatum has diverse afferent and efferent connectivity with multiple cortical, subcortical, and midbrain areas and is a key brain structure involved in reward processing and the pathophysiology of addiction (Kim, Lee, Yun, \& Kim, 2017). In addictive processes, it has been hypothesized that a ventral-to-dorsal shift of striatal control may play a critical role in habitual and then compulsive behaviors (Everitt \& Robbins, 2013). For instance, both gray matter density and white matter connectivity of the putamen were predictive of vulnerability to habitual reactions toward nolonger-rewarding outcomes (de Wit et al., 2012). The insula is closely implicated in physiological states inducing strong 
interoceptive signals of addictive urge (Droutman, Read, \& Bechara, 2015). tDCS may ameliorate online game addiction by enhancing metabolic activity in these structures. However, further studies are warranted to elucidate more detailed mechanisms underlying tDCS-induced changes of brain functions and subsequent improvement of addiction symptoms.

Some limitations should be addressed. First, since this is a single-blind study, more rigorous double-blind design is necessary for future studies. Although we used selfreport questionnaires and PET scans for the assessment, experimenter influences may not be ruled out. However, it was suggested that behavioral differences between singleblind and double-blind tDCS experiments may not be significant as expected (Thair et al., 2017). Second, whether participants noticed the stimulation type was not checked at the end of the study to ensure proper blinding. However, previous studies have reported that participants were not able to distinguish between active and sham tDCS from perceived cutaneous sensations when using the standard shamming procedure (Gandiga et al., 2006; Palm et al., 2013; Poreisz, Boros, Antal, \& Paulus, 2007). Third, multiple comparison correction was not applied in the statistical analyses and should be considered for future studies with larger samples. Fourth, this study focused on adults, and therefore caution is needed to generalize our findings to children and adolescents. Since tDCS trials in these populations are scarce and the developing brain can be more sensitive to interventions, the application of tDCS should be carefully considered only after convincing evidence is obtained from adults (Muszkat, Polanczyk, Dias, \& Brunoni, 2016). Optimization of the stimulation protocols and reassessment of efficacy and safety are required for gamers in the developmental age. Lastly, the instruments to assess self-control and motivation have not been validated for repeated evaluation within the short term.

In summary, our findings provide initial evidence that repeated tDCS to the prefrontal cortex may be beneficial for problematic online gaming in short term potentially through changes in self-control, motivation, and striatal/insular metabolism. Larger studies with longer follow-up period are warranted to confirm our findings.

Funding sources: This research was supported by the National Research Foundation of Korea (NRF) funded by the Korean government (2020R1C1C1007254, 2020R1A6A1A03043528, 2020M3E5D9080555), Institute of Information \& Communications Technology Planning \& Evaluation (2020-0-00238), and the National Institutes of Health (NIHNIMH 1R01MH111896, NIH-NINDS 1R01NS101362).

Authors' contribution: Study concept and design: H.J., I.U.S., S.H.L., Y.A.C. Data acquisition: H.J., J.K.O., E.K.C., J.J.I. Analysis and interpretation of data: H.J., S.Y., H.K., M.B., I.U.S., Y.A.C. All authors reviewed, revised, and approved the final version of the manuscript.
Conflict of interest: The City University of New York (CUNY) has IP on neurostimulation system and methods with Marom Bikson as inventor. Marom Bikson has equity in Soterix Medical Inc and serves as a consultant for Boston Scientific Inc. All other authors declare no conflict of interest.

\section{APPENDIX A: SUPPLEMENTARY MATERIAL}

The online version of this article offers supplementary material https://doi.org/10.1556/2006.2020.00092.

\section{REFERENCES}

American Psychiatric Association (2013). Diagnostic and statistical manual of mental disorders (DSM-5). Arlington, VA: American Psychiatric Association.

Bikson, M., Grossman, P., Thomas, C., Zannou, A. L., Jiang, J., Adnan, T., ..., Woods, A. J. (2016). Safety of transcranial direct current stimulation: Evidence based update 2016. Brain Stimulation, 9(5), 641-661. https://doi.org/10.1016/j.brs.2016.06.004.

Boggio, P. S., Sultani, N., Fecteau, S., Merabet, L., Mecca, T., Pascual-Leone, A., ..., Fregni, F. (2008). Prefrontal cortex modulation using transcranial DC stimulation reduces alcohol craving: A double-blind, sham-controlled study. Drug and Alcohol Dependence, 92(1-3), 55-60. https://doi.org/10.1016/j. drugalcdep.2007.06.011.

Bonelli, R. M., \& Cummings, J. L. (2007). Frontal-subcortical circuitry and behavior. Dialogues in Clinical Neuroscience, 9(2), 141-151. Retrieved from https://www.ncbi.nlm.nih.gov/ pubmed/17726913.

Carver, C. S., \& White, T. L. (1994). Behavioral inhibition, behavioral activation, and affective responses to impending reward and punishment: The BIS/BAS scales. Journal of Personality and Social Psychology, 67(2), 319.

Cho, S. S., \& Strafella, A. P. (2009). rTMS of the left dorsolateral prefrontal cortex modulates dopamine release in the ipsilateral anterior cingulate cortex and orbitofrontal cortex. PLoS One, 4(8), e6725. https://doi.org/10.1371/journal.pone.0006725.

de Wit, S., Watson, P., Harsay, H. A., Cohen, M. X., van de Vijver, I., \& Ridderinkhof, K. R. (2012). Corticostriatal connectivity underlies individual differences in the balance between habitual and goal-directed action control. Journal of Neuroscience, 32(35), 12066-12075. https://doi.org/10.1523/JNEUROSCI.1088-12.2012.

Dickler, M., Lenglos, C., Renauld, E., Ferland, F., Edden, R. A., Leblond, J., et al. (2018). Online effects of transcranial direct current stimulation on prefrontal metabolites in gambling disorder. Neuropharmacology, 131, 51-57. https://doi.org/10. 1016/j.neuropharm.2017.12.002.

Droutman, V., Read, S. J., \& Bechara, A. (2015). Revisiting the role of the insula in addiction. Trends in Cognitive Sciences, 19(7), 414-420. https://doi.org/10.1016/j.tics.2015.05.005.

Everitt, B. J., \& Robbins, T. W. (2013). From the ventral to the dorsal striatum: Devolving views of their roles in drug addiction. Neuroscience \& Biobehavioral Reviews, 37(9 Pt A), 19461954. https://doi.org/10.1016/j.neubiorev.2013.02.010. 
Fecteau, S., Fregni, F., Boggio, P. S., Camprodon, J. A., \& PascualLeone, A. (2010). Neuromodulation of decision-making in the addictive brain. Substance Use \& Misuse, 45(11), 1766-1786. https://doi.org/10.3109/10826084.2010.482434.

First MB, Spitzer RL, Gibbon M, \& Williams JBW. (2002). Structured clinical interview for DSM-IV-TR axis I disorders, research version. New York, NY: Biometrics Research, New York State Psychiatric Institute.

Fonteneau, C., Redoute, J., Haesebaert, F., Le Bars, D., Costes, N., Suaud-Chagny, M. F., et al. (2018). Frontal transcranial direct current stimulation induces dopamine release in the ventral striatum in human. Cerebral Cortex, 28(7), 2636-2646. https:// doi.org/10.1093/cercor/bhy093.

Fregni, F., Liguori, P., Fecteau, S., Nitsche, M. A., Pascual-Leone, A., \& Boggio, P. S. (2008). Cortical stimulation of the prefrontal cortex with transcranial direct current stimulation reduces cueprovoked smoking craving: A randomized, sham-controlled study. Journal of Clinical Psychiatry, 69(1), 32-40. Retrieved from https://www.ncbi.nlm.nih.gov/pubmed/18312035.

Gandiga, P. C., Hummel, F. C., \& Cohen, L. G. (2006). Transcranial DC stimulation (tDCS): A tool for double-blind shamcontrolled clinical studies in brain stimulation. Clinical Neurophysiology, 117(4), 845-850. https://doi.org/10.1016/j. clinph.2005.12.003.

Granic, I., Lobel, A., \& Engels, R. C. (2014). The benefits of playing video games. American Psychologist, 69(1), 66-78. https://doi. org/10.1037/a0034857.

Han, D. H., Bolo, N., Daniels, M. A., Arenella, L., Lyoo, I. K., \& Renshaw, P. F. (2011). Brain activity and desire for Internet video game play. Comprehensive Psychiatry, 52(1), 88-95. https://doi.org/10.1016/j.comppsych.2010.04.004.

Han, D. H., Hwang, J. W., \& Renshaw, P. F. (2010). Bupropion sustained release treatment decreases craving for video games and cue-induced brain activity in patients with Internet video game addiction. Experimental and Clinical Psychopharmacology, 18(4), 297-304. https://doi.org/10.1037/a0020023.

Hone-Blanchet, A., Edden, R. A., \& Fecteau, S. (2016). Online effects of transcranial direct current stimulation in real time on human prefrontal and striatal metabolites. Biological Psychiatry, 80(6), 432-438. https://doi.org/10.1016/j.biopsych. 2015.11.008.

Hong, S. B., Harrison, B. J., Dandash, O., Choi, E. J., Kim, S. C., Kim, H. H., ..., Yi, S. H. (2015). A selective involvement of putamen functional connectivity in youth with internet gaming disorder. Brain Research, 1602, 85-95. https://doi.org/10.1016/j. brainres.2014.12.042.

Hong, H., Kim, H., Kim, J., \& Kim, J. (2012). Validity and reliability validation of the Korean version of the Brief Self-Control Scale (BSCS). Korean Journal of Psychology: General, 31(4), 1193-1210.

Ho, R. C., Zhang, M. W., Tsang, T. Y., Toh, A. H., Pan, F., Lu, Y., ..., Mak, K. K. (2014). The association between internet addiction and psychiatric co-morbidity: A meta-analysis. BMC Psychiatry, 14, 183. https://doi.org/10.1186/1471-244X-14-183.

Im, J. J., Jeong, H., Bikson, M., Woods, A. J., Unal, G., Oh, J. K., ..., Chung, Y. A. (2019). Effects of 6-month at-home transcranial direct current stimulation on cognition and cerebral glucose metabolism in Alzheimer's disease. Brain Stimulation, 12(5), 1222-1228. https://doi.org/10.1016/j.brs.2019.06.003.
Keeser, D., Meindl, T., Bor, J., Palm, U., Pogarell, O., Mulert, C., ..., Padberg, F. (2011). Prefrontal transcranial direct current stimulation changes connectivity of resting-state networks during fMRI. Journal of Neuroscience, 31(43), 15284-15293. https://doi.org/10.1523/JNEUROSCI.0542-11.2011.

Kekic, M., Boysen, E., Campbell, I. C., \& Schmidt, U. (2016). A systematic review of the clinical efficacy of transcranial direct current stimulation (tDCS) in psychiatric disorders. Journal of Psychiatric Research, 74, 70-86. https://doi.org/10.1016/j. jpsychires.2015.12.018.

Kim, Y., Jeong, J. E., Cho, H., Jung, D. J., Kwak, M., Rho, M. J., ..., Choi, I. Y. (2016). Personality factors predicting smartphone addiction predisposition: Behavioral inhibition and activation systems, impulsivity, and self-control. PLoS One, 11(8), e0159788. https://doi.org/10.1371/journal.pone.0159788.

Kim, K., \& Kim, W. S. (2001). Korean-BAS/BIS scale. Korean Journal of Health Psychology, 6(2), 19-37.

Kim, H., Kim, Y. K., Lee, J. Y., Choi, A. R., Kim, D. J., \& Choi, J. S. (2019). Hypometabolism and altered metabolic connectivity in patients with internet gaming disorder and alcohol use disorder. Progress In Neuro-Psychopharmacology \& Biological Psychiatry, 95, 109680. https://doi.org/10.1016/j.pnpbp.2019.109680.

Kim, H. J., Lee, J. H., Yun, K., \& Kim, J. H. (2017). Alterations in striatal circuits underlying addiction-like behaviors. Molecules and Cells, 40(6), 379-385. https://doi.org/10.14348/molcells. 2017.0088.

King, D. L., Delfabbro, P. H., Wu, A. M. S., Doh, Y. Y., Kuss, D. J., Pallesen, S., ..., Sakuma, H. (2017). Treatment of Internet gaming disorder: An international systematic review and CONSORT evaluation. Clinical Psychology Review, 54, 123-133. https://doi.org/10.1016/j.cpr.2017.04.002.

Kober, H., Mende-Siedlecki, P., Kross, E. F., Weber, J., Mischel, W., Hart, C. L., et al. (2010). Prefrontal-striatal pathway underlies cognitive regulation of craving. Proceedings of the National Academy of Sciences of the United States of America, 107(33), 14811-14816. https://doi.org/10.1073/pnas.1007779107.

Ko, C. H., Yen, J. Y., Yen, C. F., Chen, C. S., Weng, C. C., \& Chen, C. C. (2008). The association between internet addiction and problematic alcohol use in adolescents: The problem behavior model. CyberPsychology and Behavior, 11(5), 571-576. https:// doi.org/10.1089/cpb.2008.0199 10.1089/cpb.2007.0199.

Kuss, D. J., Griffiths, M. D., \& Pontes, H. M. (2017). Chaos and confusion in DSM-5 diagnosis of Internet Gaming Disorder: Issues, concerns, and recommendations for clarity in the field. Journal of Behavioral Addictions, 6(2), 103-109. https://doi.org/ 10.1556/2006.5.2016.062.

Kwon, Y. H., \& Jang, S. H. (2012). Onsite-effects of dual-hemisphere versus conventional single-hemisphere transcranial direct current stimulation: A functional MRI study. Neural Regeneration Research, 7(24), 1889-1894. https://doi.org/10. 3969/j.issn.1673-5374.2012.24.007.

Lee, H. W., Choi, J. S., Shin, Y. C., Lee, J. Y., Jung, H. Y., \& Kwon, J. S. (2012). Impulsivity in internet addiction: A comparison with pathological gambling. Cyberpsychology, Behavior, and Social Networking, 15(7), 373-377. https://doi.org/10.1089/cyber.2012. 0063.

Lee, S. H., Im, J. J., Oh, J. K., Choi, E. K., Yoon, S., Bikson, M., ..., Chung, Y. A. (2018). Transcranial direct current stimulation for 
online gamers: A prospective single-arm feasibility study. Journal of Behavioral Addictions, 7(4), 1166-1170. https://doi. org/10.1556/2006.7.2018.107.

Lee, S. H., Im, J. J., Oh, J. K., Choi, E. K., Yoon, S., Bikson, M., ..., Chung, Y. A. (2019). Transcranial direct current stimulation for online gamers. Journal of Visualized Experiments(153). https:// doi.org/10.3791/60007.

Lee, K., Lee, H.-K., Gyeong, H., Yu, B., Song, Y.-M., \& Kim, D. (2013). Reliability and validity of the Korean version of the Internet Addiction Test among college students. Journal of Korean Medical Science, 28(5), 763-768.

Lindenberg, R., Nachtigall, L., Meinzer, M., Sieg, M. M., \& Floel, A. (2013). Differential effects of dual and unihemispheric motor cortex stimulation in older adults. Journal of Neuroscience, 33(21), 9176-9183. https://doi.org/10.1523/JNEUROSCI.005513.2013 .

Loftus, A. M., Yalcin, O., Baughman, F. D., Vanman, E. J., \& Hagger, M. S. (2015). The impact of transcranial direct current stimulation on inhibitory control in young adults. Brain and Behavior, 5(5), e00332. https://doi.org/10.1002/brb3.332.

Loo, C. K., Sachdev, P., Martin, D., Pigot, M., Alonzo, A., Malhi, G. S., ..., Mitchell, P. (2010). A double-blind, sham-controlled trial of transcranial direct current stimulation for the treatment of depression. The International Journal of Neuropsychopharmacology, 13(1), 61-69. https://doi.org/10.1017/ S1461145709990411.

Muszkat, D., Polanczyk, G. V., Dias, T. G., \& Brunoni, A. R. (2016). Transcranial direct current stimulation in child and adolescent psychiatry. Journal of Child and Adolescent Psychopharmacology, 26(7), 590-597. https://doi.org/10.1089/cap.2015.0172.

Na, E., Lee, H., Choi, I., \& Kim, D. J. (2017). Comorbidity of internet gaming disorder and alcohol use disorder: A focus on clinical characteristics and gaming patterns. American Journal on Addictions, 26(4), 326-334. https://doi.org/10.1111/ajad. 12528.

Nitsche, M. A., Cohen, L. G., Wassermann, E. M., Priori, A., Lang, N., Antal, A., ..., Pascual-Leone, A. (2008). Transcranial direct current stimulation: State of the art 2008. Brain Stimulation, 1(3), 206-223. https://doi.org/10.1016/j.brs.2008.06.004.

Ohmann, H. A., Kuper, N., \& Wacker, J. (2018). Left frontal anodal tDCS increases approach motivation depending on reward attributes. Neuropsychologia, 119, 417-423. https://doi.org/10. 1016/j.neuropsychologia.2018.09.002.

Palm, U., Reisinger, E., Keeser, D., Kuo, M. F., Pogarell, O., Leicht, G., ..., Padberg, F. (2013). Evaluation of sham transcranial direct current stimulation for randomized, placebo-controlled clinical trials. Brain Stimulation, 6(4), 690-695. https://doi.org/ 10.1016/j.brs.2013.01.005.

Palm, U., Schiller, C., Fintescu, Z., Obermeier, M., Keeser, D., Reisinger, E., ..., Padberg, F. (2012). Transcranial direct current stimulation in treatment resistant depression: A randomized double-blind, placebo-controlled study. Brain Stimulation, 5(3), 242-251. https://doi.org/10.1016/j.brs.2011.08.005.

Pawlikowski, M., \& Brand, M. (2011). Excessive internet gaming and decision making: Do excessive world of Warcraft players have problems in decision making under risky conditions? Psychiatry Research, 188(3), 428-433. https://doi.org/10.1016/j. psychres.2011.05.017.
Pena-Gomez, C., Sala-Lonch, R., Junque, C., Clemente, I. C., Vidal, D., Bargallo, N., ..., Bartres-Faz, D. (2012). Modulation of largescale brain networks by transcranial direct current stimulation evidenced by resting-state functional MRI. Brain Stimulation, 5(3), 252-263. https://doi.org/10.1016/j.brs.2011.08.006.

Poreisz, C., Boros, K., Antal, A., \& Paulus, W. (2007). Safety aspects of transcranial direct current stimulation concerning healthy subjects and patients. Brain Research Bulletin, 72(4-6), 208214. https://doi.org/10.1016/j.brainresbull.2007.01.004.

Reynolds, B., Penfold, R. B., \& Patak, M. (2008). Dimensions of impulsive behavior in adolescents: Laboratory behavioral assessments. Experimental and Clinical Psychopharmacology, 16(2), 124-131. https://doi.org/10.1037/1064-1297.16.2.124.

Rudroff, T., Workman, C. D., Fietsam, A. C., \& Ponto, L. L. B. (2020). Imaging transcranial direct current stimulation (tDCS) with positron emission tomography (PET). Brain Sciences, 10(4). https://doi.org/10.3390/brainsci10040236.

Sauvaget, A., Trojak, B., Bulteau, S., Jimenez-Murcia, S., FernandezAranda, F., Wolz, I., ..., Grall-Bronnec, M. (2015). Transcranial direct current stimulation (tDCS) in behavioral and food addiction: A systematic review of efficacy, technical, and methodological issues. Frontiers in Neuroscience, 9, 349. https:// doi.org/10.3389/fnins.2015.00349.

Soyata, A. Z., Aksu, S., Woods, A. J., Iscen, P., Sacar, K. T., \& Karamursel, S. (2019). Effect of transcranial direct current stimulation on decision making and cognitive flexibility in gambling disorder. European Archives of Psychiatry and Clinical Neuroscience, 269(3), 275-284. https://doi.org/10.1007/ s00406-018-0948-5.

Stagg, C. J., Best, J. G., Stephenson, M. C., O’Shea, J., Wylezinska, M., Kincses, Z. T., et al. (2009). Polarity-sensitive modulation of cortical neurotransmitters by transcranial stimulation. Journal of Neuroscience, 29(16), 5202-5206. https://doi.org/10.1523/ JNEUROSCI.4432-08.2009.

Strafella, A. P., Paus, T., Barrett, J., \& Dagher, A. (2001). Repetitive transcranial magnetic stimulation of the human prefrontal cortex induces dopamine release in the caudate nucleus. Journal of Neuroscience, 21(15), RC157. Retrieved from https://www. ncbi.nlm.nih.gov/pubmed/11459878.

Takeuchi, H., Taki, Y., Hashizume, H., Asano, K., Asano, M., Sassa, Y., ..., Kawashima, R. (2016). Impact of videogame play on the brain's microstructural properties: Cross-sectional and longitudinal analyses. Molecular Psychiatry, 21(12), 1781-1789. https://doi.org/10.1038/mp.2015.193.

Tangney, J. P., Baumeister, R. F., \& Boone, A. L. (2004). High selfcontrol predicts good adjustment, less pathology, better grades, and interpersonal success. Journal of Personality, 72(2), 271324. Retrieved from https://www.ncbi.nlm.nih.gov/pubmed/ 15016066.

Thair, H., Holloway, A. L., Newport, R., \& Smith, A. D. (2017). Transcranial Direct Current Stimulation (tDCS): A beginner's guide for design and implementation. Frontiers in Neuroscience, 11, 641. https://doi.org/10.3389/fnins.2017.00641.

Tian, M., Chen, Q., Zhang, Y., Du, F., Hou, H., Chao, F., et al. (2014). PET imaging reveals brain functional changes in internet gaming disorder. European Journal of Nuclear Medicine and Molecular Imaging, 41(7), 1388-1397. https://doi.org/ 10.1007/s00259-014-2708-8. 
Tsitsika, A., Critselis, E., Janikian, M., Kormas, G., \& Kafetzis, D. A. (2011). Association between internet gambling and problematic internet use among adolescents. Journal of Gambling Studies, 27(3), 389-400.

Van Rooij, A. J., Kuss, D. J., Griffiths, M. D., Shorter, G. W., Schoenmakers, M. T., \& D, V. D. M. (2014). The (co-)occurrence of problematic video gaming, substance use, and psychosocial problems in adolescents. Journal of Behavioral Addictions, 3(3), 157-165. https://doi.org/10.1556/JBA.3.2014.013.

Weinstein, A. M. (2017). An update overview on brain imaging studies of internet gaming disorder. Frontiers in Psychiatry, 8, 185. https://doi.org/10.3389/fpsyt.2017.00185.

Wiers, C. E., Cabrera, E., Skarda, E., Volkow, N. D., \& Wang, G. J. (2016). PET imaging for addiction medicine: From neural mechanisms to clinical considerations. Progress in Brain Research, 224, 175-201. https://doi.org/10.1016/bs.pbr.2015.07.016.

Wolfling, K., Muller, K. W., Dreier, M., Ruckes, C., Deuster, O., Batra, A., et al. (2019). Efficacy of short-term treatment of internet and computer game addiction: A randomized clinical trial. JAMA Psychiatry, 76(10), 1018-1025. https://doi.org/10. 1001/jamapsychiatry.2019.1676.

Yao, Y. W., Liu, L., Ma, S. S., Shi, X. H., Zhou, N., Zhang, J. T., et al. (2017). Functional and structural neural alterations in internet gaming disorder: A systematic review and meta-analysis. Neuroscience \& Biobehavioral Reviews, 83, 313-324. https://doi. org/10.1016/j.neubiorev.2017.10.029.

Yen, J. Y., Ko, C. H., Yen, C. F., Chen, C. S., \& Chen, C. C. (2009). The association between harmful alcohol use and internet addiction among college students: Comparison of personality. Psychiatry and Clinical Neurosciences, 63(2), 218-224. https:// doi.org/10.1111/j.1440-1819.2009.01943.x.

Young, K. S. (1998). Caught in the net: How to recognize the signs of Internet addiction. New York: John Wiley \& Sons, Inc.

Yun, K., Song, I. U., \& Chung, Y. A. (2016). Changes in cerebral glucose metabolism after 3 weeks of noninvasive electrical stimulation of mild cognitive impairment patients. Alzheimer's Research \& Therapy, 8(1), 49. https://doi.org/10.1186/s13195016-0218-6. 